\title{
The Gattini cameras for optical sky brightness measurements in Antarctica
}

\author{
Anna Moore* $^{* \mathrm{a}}$, Eric Aristidi ${ }^{\mathrm{b}}$, Michael Ashley ${ }^{\mathrm{c}}$, Maurizio Busso ${ }^{\mathrm{d}}$, Maurizio Candidi ${ }^{\mathrm{d}}$, Jon Everett ${ }^{\mathrm{c}}$, \\ Suzanne Kenyon ${ }^{c}$, Jon Lawrence ${ }^{c}$, D, Luong-Van ${ }^{c}$, Andre Phillips ${ }^{c}$, Brice Le Roux ${ }^{\mathrm{e}}$, Roberto \\ Ragazzoni $^{\mathrm{e}}$, Piero Salinari ${ }^{\mathrm{e}}$, John Storey ${ }^{\mathrm{c}}$, Melinda Taylor ${ }^{\mathrm{c}}$, Gino Tosti ${ }^{\mathrm{d}}$, Tony Travouillon ${ }^{\mathrm{f}}$ \\ ${ }^{a}$ California Institute of Technology, Pasadena, CA 91106, USA \\ ${ }^{b}$ LUAN, Université de Nice, Parc Valrose, 06108 Nice Cedex 2, France \\ ${ }^{c}$ School of Physics, University of New South Wales, Sydney, NSW 2052, Australia \\ Dipartimento di Fisica, Università di Perugia, via A. Pascoli, 06123 Perugia, Italy \\ ${ }^{e}$ Istituto di Fisica dello Spazio, CNR, via Fosso Cavaliere 100, Roma, and Programma Nazionale di \\ Ricerche in Antartide, ENEA, Roma, Italy \\ ${ }^{f}$ Osservatorio Astrofisico di Arcetri, Largo Enrico Fermi, 5, Firenze 50125, Italy \\ ${ }^{\mathrm{g}}$ TMT project 102-08, California Institute of Technology, Pasadena, CA 91106, USA
}

\begin{abstract}
The Gattini cameras are two site testing instruments for the measurement of optical sky brightness, large area cloud cover and auroral detection of the night sky above the high altitude Dome $\mathrm{C}$ site in Antarctica. The cameras have been in operation since January 2006. The cameras are transit in nature and are virtually identical, both adopting Apogee Alta ccd detectors. The camera called Gattini-SBC images a 6 degree field centred on the South Pole, an elevation of $75^{\circ}$ at the Dome C site. The camera takes repeated images of the same 6 degree field in the Sloan g' band (centred on 477nm) and, by adopting a lens with sufficiently long focal length, one can integrate the sky background photons and directly compare to the equivalent values of the stars within the field. The second camera, called Gattini-allsky, incorporates a fish-eye lens and images $\sim 110$ degree field centred on local zenith. By taking frequent images of the night sky we will obtain long term cloud cover statistics, measure the sky background intensity as a function of solar and lunar altitude and phase and directly measure the spatial extent of bright aurora if present and when they occur. An overview of the project is presented together with preliminary results from data taken since operation of the cameras in January 2006.
\end{abstract}

\section{INTRODUCTION}

Since the release of spectacular seeing measurements at visible wavelengths $(550 \mathrm{~nm})$ taken at the Antarctic site of Dome $\mathrm{C}$ (location shown in Figure 1 right) during summertime at ground level [1] and during winter-time [2] there have been several proposals for $2 \mathrm{~m}$ class optical telescopes that wish to exploit this unique location [3], [4], [5]. Optical sky background measurements, that include twilight and aurora, have largely been unquantified for this site. Given the latitude of Dome C $\left(-75^{\circ}\right)$ the site experiences short periods of twilight diurnally throughout the winter season, unlike the South Pole that undergoes an extended period of true astronomical dark time (defined by a solar altitude less than $-18^{\circ}$ ). However, there is evidence that the aerosol content above the Dome $\mathrm{C}$ site is extremely low. As broadband scattering from aerosol particles is a contributing factor to increased sky brightness at visible wavelengths in particular, a reduction in sky brightness could result for particular target observing angles. An estimate of the true amount of dark time experienced above this site is important for any proposing optical facility. An excellent summary of the optical sky brightness modelled by constituent part for the Dome $\mathrm{C}$ site can be found in [6].

* email to: amoore@astro.caltech.edu

Ground-based and Airborne Telescopes, edited by Larry M. Stepp, Proc. of SPIE

Vol. 6267, 62671N, (2006) - 0277-786X/06/\$15 - doi: 10.1117/12.672525

Proc. of SPIE Vol. $626762671 \mathrm{~N}-1$ 

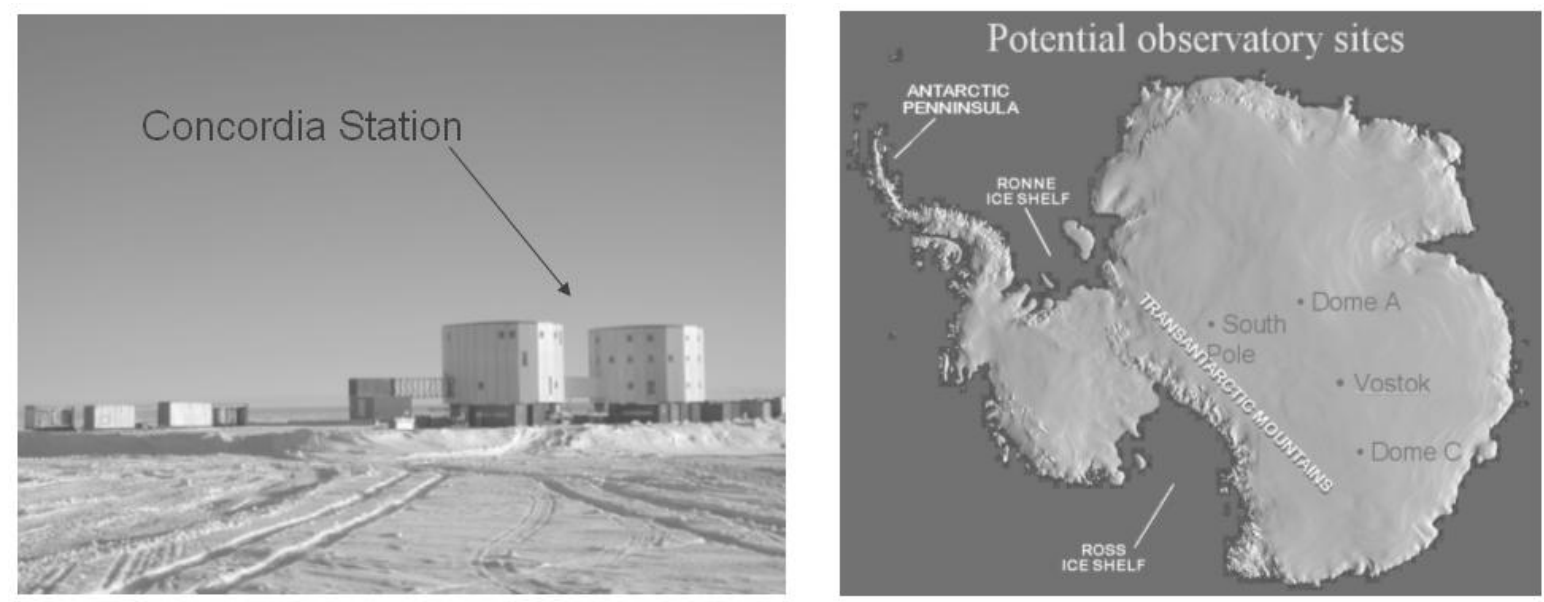

Figure 1: The French/Italian Concordia installation at the Dome C site provides comfortable habitation and work environment for staff throughout the year (left); Relative locations of various high altitude sites including Dome C (right)

The GATTINI cameras were designed to provide long term sky brightness statistics for the sky above Dome C. The 2 cameras are located on the roof of Atmospheric laboratory (shown in Figure 2), approximately $1 \mathrm{~km}$ from French/Italian Concordia station shown in Figure 1 (left).

The cameras, both transit in nature, will also provide a method to:

- Calculate optical sky brightness as a function of lunar phase, altitude and distance to target

- Calculate cloud cover across a large area of sky

- Detect spatially bright auroral events if and when they occur

- Estimate the optical extinction

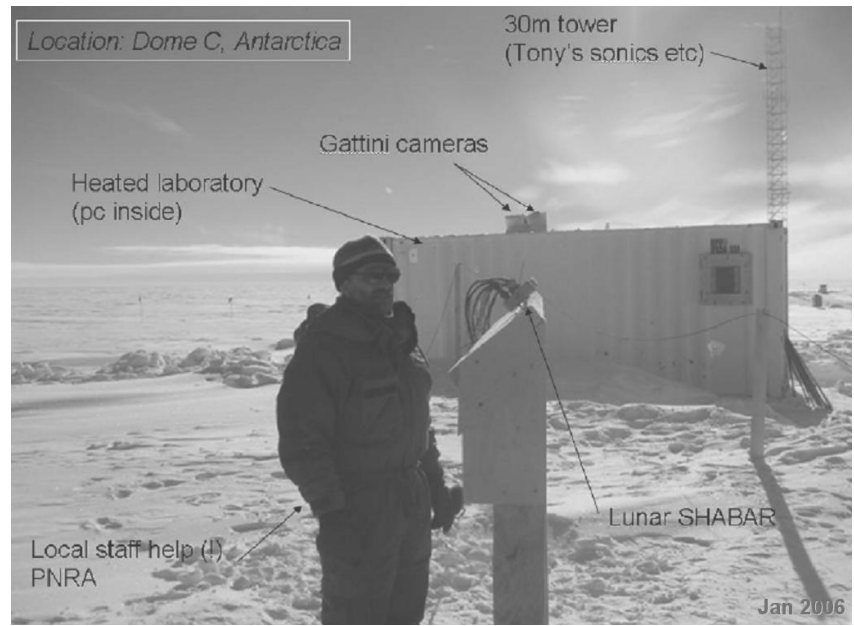

Figure 2: The Gattini cameras are located on the roof of the atmospheric laboratory at the Dome C site as shown above. The laboratory is a heated environment, a requirement for the control computer. The $30 \mathrm{~m}$ tower shown in the background is used for ground layer profiling. In the foreground a transit lunar shabar points northwards.

\section{GATTINI-SBC}

Gattini-SBC, shown in Figure 3, is a camera that images a $6^{\circ}$ by $4^{\circ}$ field of view centred close to the South Pole (zenith angle of $\sim 15^{\circ}$ at the Dome $\mathrm{C}$ site). The primary goal is to measure accurately the contribution to the optical sky background by integrating sky photons in between the resolved stars on the CCD. 
In summary the Gattini-SBC:

- Images a field $6^{\circ}$ by $4^{\circ}$ centered on the SP every 20 minutes

- $\quad 40 \mathrm{sec}$ exp time - no star trails (though not mandatory)

- Identify bright stars and use for flux calibration of sky pixel intensity

- Produces a 2D sky brightness map every 20 minutes

- Side project- variable star monitoring and searching for planetary transits

The primary aim of Gattini-SBC is the accurate measurement of sky background magnitude at optical wavelengths above the Dome $\mathrm{C}$ site. To be able to integrate sky background photons without stellar contamination the plate scale must be small enough such that the stellar density on the CCD is small but not small enough that the individual stars cannot be identified and removed. Identification is essential as it provides a direct calibration source for the sky background photons. To aid greatly in this task the FoV of the camera is centred roughly on the South Pole.

A Nikor $\mathrm{f} / 135 \mathrm{~mm}$ lens was chosen resulting in a plate scale of $\sim 11 \mathrm{arcsec} /$ pix. Set a maximum stop diameter, the aperture of the Gattini-SBC camera is $\sim 50 \mathrm{~mm}(\mathrm{~F} / 2.8)$. We have chosen to image through a sloan g' filter that is attached to the front of the lens for meaningful comparison to other sites (477nm central wavelength). A raw image taken with Gattini$\mathrm{SBC}$ is shown in Figure 4.

\section{GATTINI-ALLSKY}

The second instrument is a wide field camera with a primary goal of measuring accurate, frequent and long term cloud statistics at the Dome $\mathrm{C}$ site as well as the recording of bright auroral events if and when they occur. The camera, shown in Figure 5, uses a fish-eye lens that in combination with the camera images a field of view $110^{\circ}$ by $90^{\circ}$ roughly centred on local zenith. A raw image taken with the camera is shown in Figure 6.

\section{PROJECT TIMELINE}

A brief history of the project as follows:

- Funded by INAF at 30k Euro level for 3 Gattini instruments at INAF:Arcetri (Nov 2004)

- Initial design and testing of Apogee cameras at INAF:Arcetri

- Assembly and low-temperature testing performed at UNSW during 2005 (Feb-April, Aug)

- On-site test ( 1 month) at Siding Spring Observatory (Nov 2005)

- $\quad$ Packed and shipped from UNSW in Dec 2006

- Arrival at Dome C via PNRA Jan 2006 (Hercules from Christchurch)

- Installed at Dome C by Tony Travouillon, Eric Aristidi and help from Concordia station staff in Jan 2006

- Both cameras operating since Feb 2006, Eric Aristidi is winter-over astronomer

\section{CAMERA DESIGN}

\subsection{Apogee CCD}

Each camera uses an identical Apogee Alta U2020 interline camera is supplied with an internal Peltier cooler. The cameras are USB interface (Ethernet versions are available, though not at the time). The CCD chip is a Kodak KAI2020 with 1600 by 12007.4 micron pixels. The interline feature is very important as the system requires no mechanical shutter. The CCD has a QE of $\sim 40 \%$. The Peltier cooler is rarely used for winter-time observations and usually remains at a constant $30 \%$ power level for both cameras. The Apogee Alta cameras, as shown in Figure 7 prior to assembly, have shown to be extremely reliable. 

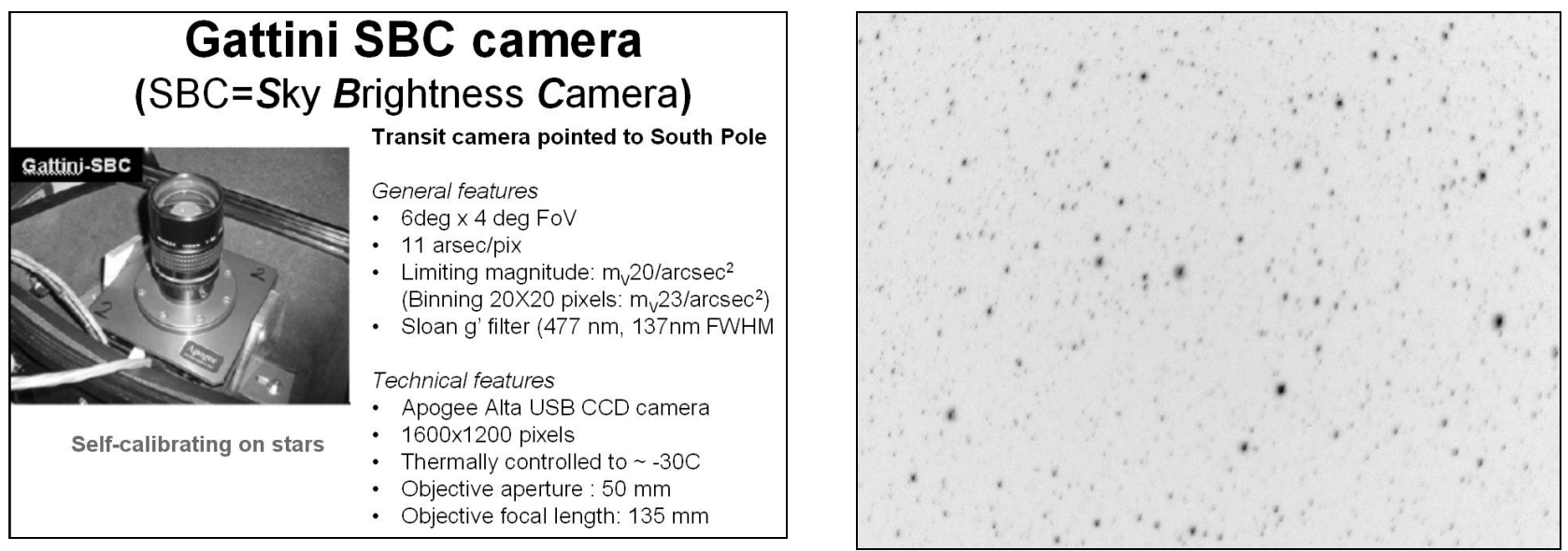

Figure 3: (left) A summary of the Gattini-SBC camera (Sky Brightness Camera) shown inside the enclosure (left). The camera measures the sloan g' $(477 \mathrm{~nm})$ sky background intensity every 20 minutes at the Dome C site. Accurate calibration across the field of view is performed the stars in the field. The camera objective lens is a Nikor $\mathrm{f} / 135 \mathrm{~mm}$

$\mathrm{F} / 2.8$ set at maximum stop diameter.

Figure 4: (right) Raw (negative) image taken with the Gattini-SBC camera. The image shows a $6^{\circ}$ by $4^{\circ}$ rectangular field centred on the South Pole. Integration time $=40 \mathrm{~s}$. The faintest magnitude star detected is equivalent to $\sim 21 \mathrm{Vmag} / \operatorname{arcsec}^{2}$, binning of small pixel areas is required to reach fainter sky background measurements. Image taken April 8th 2006 at 23:48 local time

\subsection{Mechanical Design}

The mechanical housings for the cameras are virtually identical. The enclosure comprises a $3 \mathrm{~mm}$ thick plastic box large enough to fit the camera, optics and remaining components, as shown in Figure 8. The windows for each camera are different. For Gattini-allsky, a $1 / 8^{\text {th }}$ " thick acrylic dome is bonded to the enclosure to allow suitable viewing of the night sky with negligible transmission loss. For Gattini-SBC the acrylic dome offered too much optical aberration therefore a flat Pyrex glass window was chosen instead. The windows are the only difference between the 2 enclosures.

Each enclosure is sealed air-tight as best as possible using Silastic (a rubber sealant) at every joint and a fluorosilicone oring between the lid and main section. To prevent a large pressure difference forming across the enclosure wall a Brownwell valve is incorporated into the side of each box, allowing air to pass in both directions if the pressure difference increases above $\sim 1$ atm. Polyethylene insulation lines the walls of the interior of the boxes while special fibre glass insulation is used on the outside. Finally a reflective layer covers both enclosures to reflect sunlight and prevent excessive heating of the enclosures when the sun is above the horizon.

A powerful desiccant is placed inside each enclosure to remove water in the air $\left(\mathrm{CaH}_{2}\right)$ to help prevent ice formation on the inside of the windows. By keeping the internal temperature of the enclosures a few degrees above ambient we aim to prevent ice and/or snow forming on the outside surface of the window. 

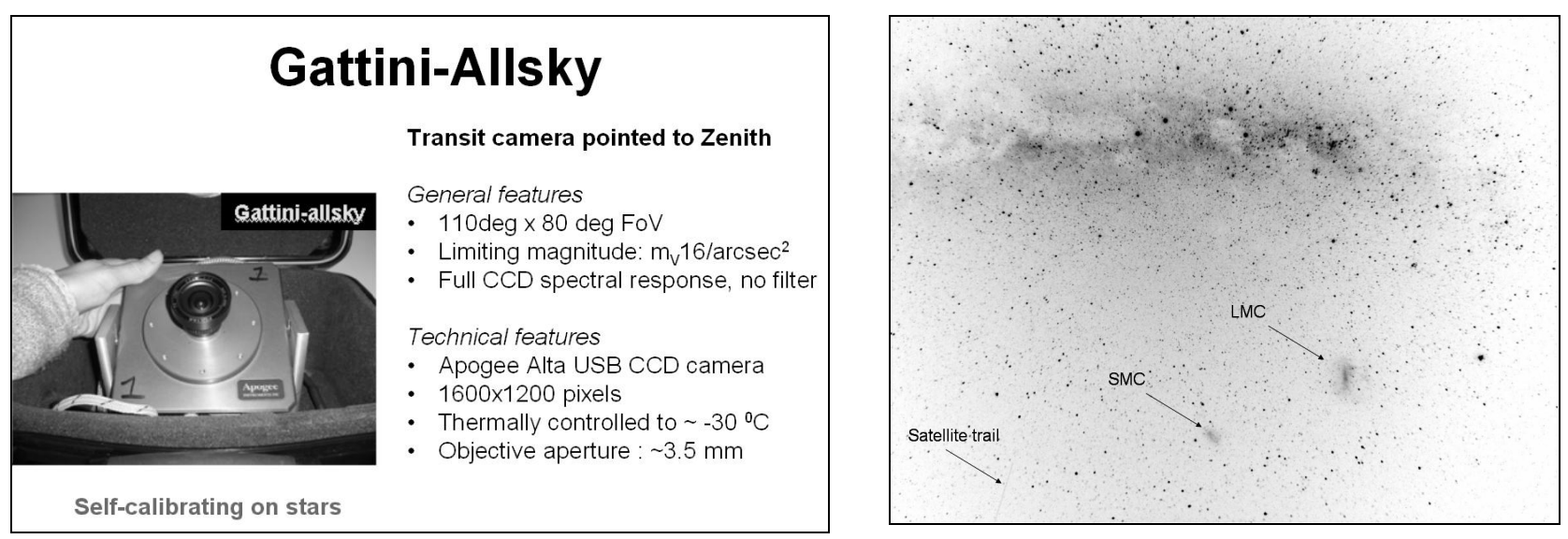

Figure 5: (left) A summary of the Gattini-allsky camera (shown left). The camera acquires broadband images of a large area field centred at local zenith at the Dome $\mathrm{C}$ site. No filter is included in the system. The lens is a Pentax fish-eye $\mathrm{f} / 6 \mathrm{~mm} \mathrm{~F} / 1.4$ with the stop setting at maximum diameter.

Figure 6: (right) A raw (negative) image taken by the Gattini-allsky camera. The image has not been corrected for distortion nor flat field effects (both are significant effects for a fish-eye objective). Significant features are highlighted in the image. The 40s exposure was taken on March 29th 2006 during dark time.

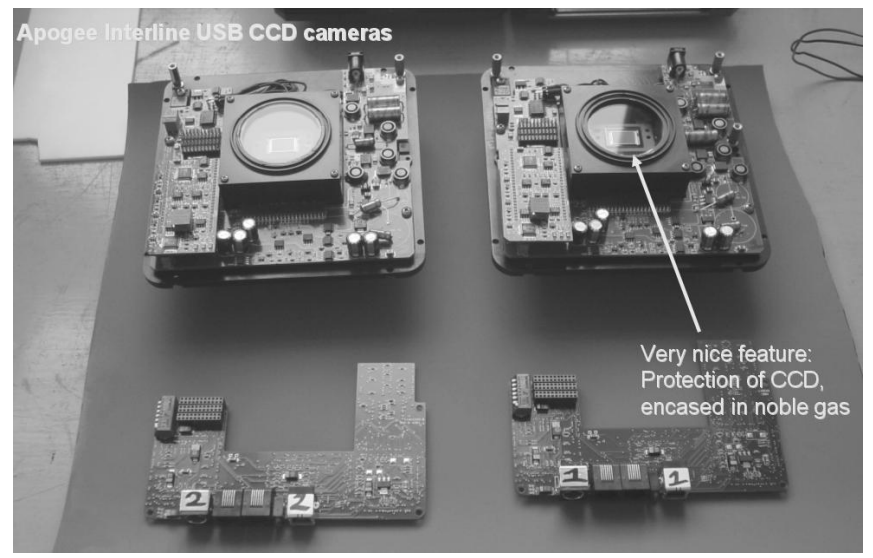

Figure 7: The innards of the Apogee CCD cameras. The Alta USB cameras are identical and contain a Kodak 1600 by 1200 interline chip, so not requiring a mechanical shutter.

\subsection{Thermal Design}

The thermal design was approached in the following simple way:

1. Place each camera inside a heated enclosure (man-made seeing is not an issue for both cameras given the pixel sampling on the sky is large)

2. Keep the enclosure as airtight as possible

3. Maintain the inside temperature to at least a few degrees warmer than ambient to keeps prevent ice and/or snow forming on the outside of the window

4. Incorporate a high pressure release valve

5. Include a strong (unfortunately probably highly toxic) $\mathrm{CaH}_{2}$ desiccant inside enclosure to remove water from air keeps ice from forming on inside of window 

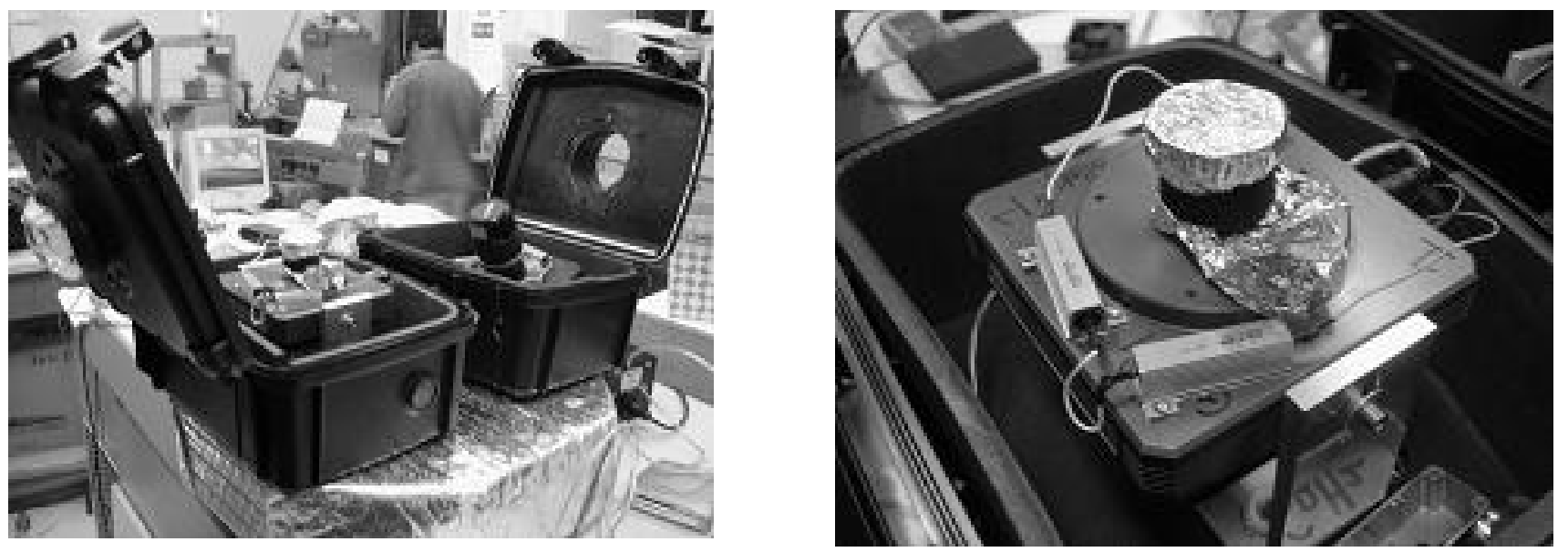

Figure 8: (left) Each camera is located inside a small enclosure that is heated to a constant temperature of $-10^{\circ} \mathrm{C}$. At this temperature the cameras can be power cycled successfully. Turbulence produced by the enclosures being substantially warmer than ambient does not affect the measurements made by the cameras.

Figure 9: (right) Two DC heaters are thermally coupled to the body of each camera. Power switching of the heaters provides the thermal control of the enclosure temperature.

Heat is provided by two sources (1) the heat from the Apogee CCD Peltier cooler and (2) two DC heaters that are thermally bonded to the metal housing of each Apogee camera, as shown in Figure 9. Using heat from the Peltier cooler in this way is not ideal (rather than removing it from the enclosure) because it can lead to a "runaway" heating effect and often did during the summer months when the sun was high and the ambient temperature (relatively) high. However, the Peltier cooler does provide a useful source of heat and provides automatically fans for circulation of the air inside the enclosure. Added to this is the complexity of removing the heat from the Peltier.

\subsection{Cabling}

Low temperature rated Teflon coated cable was used throughout the design of the cabling. In addition to this, Milspec crimp connectors were used wherever possible. Soldered joints, non low temperature rated cable and USB connectors were avoided in particular due to their low reliability. Specific attention was paid to grounding as the high Antarctic site is particularly prone to static build-up.

\section{AUTOMATED CONTROL}

The cameras are controlled by an industrial pc-104 system based on the supervisor system used by UNSW for the control of the AASTINO and corresponding instruments [7]. The $300 \mathrm{MHz}$ low power consumption pc saves critical data to $8 \mathrm{~GB}$ solid state flashdisk space that also contains the Linux operating system. Two 200GB standard rotary hard disk drives provide additional high volume disk space. All data is backed up to the Concordia station once per week. Power supply to the pc-104 and hence to the entire system (including the cameras) comes from the Concordia station AC mains power. Except for external AC power the Gattini system is designed to be as independent as possible.

A UPS is included in the system and provides what has become a critical function. When there is a drop of station power the UPS provides sufficient power for the Gattini system to power down before going into hibernation. This is particularly important for processes such as hard disk writes that can corrupt a disk drive if stopped mid-cycle. The Gattini control system is shown in Figure 10 with a blow-up of the electronics rack shown in Figure 11. 

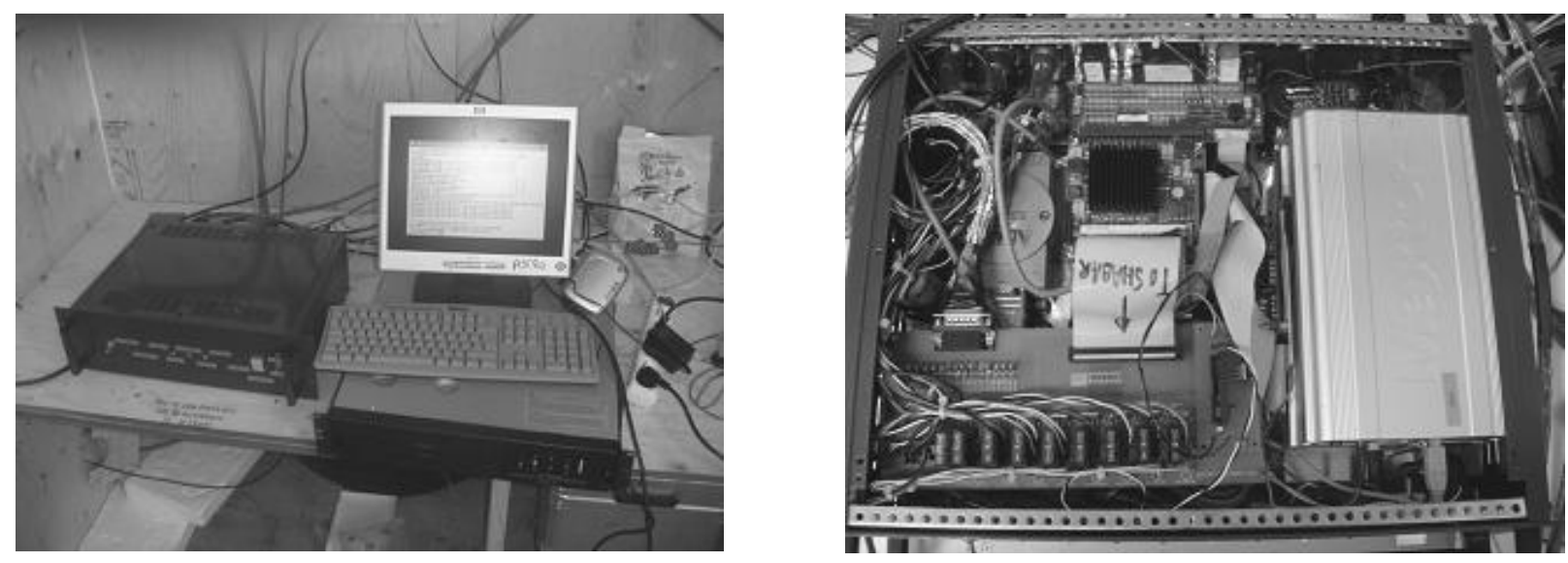

Figure 10: (left) The Supervisor computer of the Gattini cameras provides fully automated control of the instruments during the year, including temperature monitoring and Iridium connection to Sydney for data and status download. A UPS is included to provide a safe method for shutdown if and when station power recycles.

Figure 11: (right) The computer is a low power industrial grade PC104 stackable system located in a standard 19" rack. The hard disk drives and power switching electronics are shown.

\section{DATA MANAGEMENT}

Data storage, transfer and communications are largely handled by the UNSW team in Sydney. A NSF supplied Iridium link enables direct connection to the Gattini supervisor computer. On a day to day basis the process of retrieving system status updates and up to $1 \mathrm{MB}$ of data per connection is automated, however, it is possible to change any feature of the system throughout the winter season. This is extremely useful for changing variables such as camera exposure time and cadence during the season when useful data is first retrieved. A LAN connection between the Gattini PC and Concordia station means that an automated back-up can occur, currently set to once per week.

The transfer of system status data throughout the season has been a critical feature so far. A sample of this data is shown in Figure 12.

\section{THERMAL TESTING}

The Gattini cameras, together with their respective enclosures, were thermally cycled for a substantial period of time using the large biological freezer in the UNSW labs. The testing was extremely important in proving the viability of the mechanical enclosure, the design of the thermal management and testing of the Apogee cameras at a reasonable low temperature. Several additions were made to the original design of the system directly because of the thermal testing, a portion of which is shown in Figure 13- Figure 15.

\section{SITE TESTING}

After assembly in UNSW labs, the Gattini cameras were shipped to Siding Springs Observatory for a period of $\sim 1$ month. The site test provided a very useful test for software and automated control in particular.

\section{LESSONS LEARNT}

Likely due to substantial pre-testing, both low temperature and site testing, thankfully there are few negatives to report. The components that are working particularly well are:

- Heating system

- Data transfer (UNSW/NSF Iridium card)

- Apogee camera reliability 


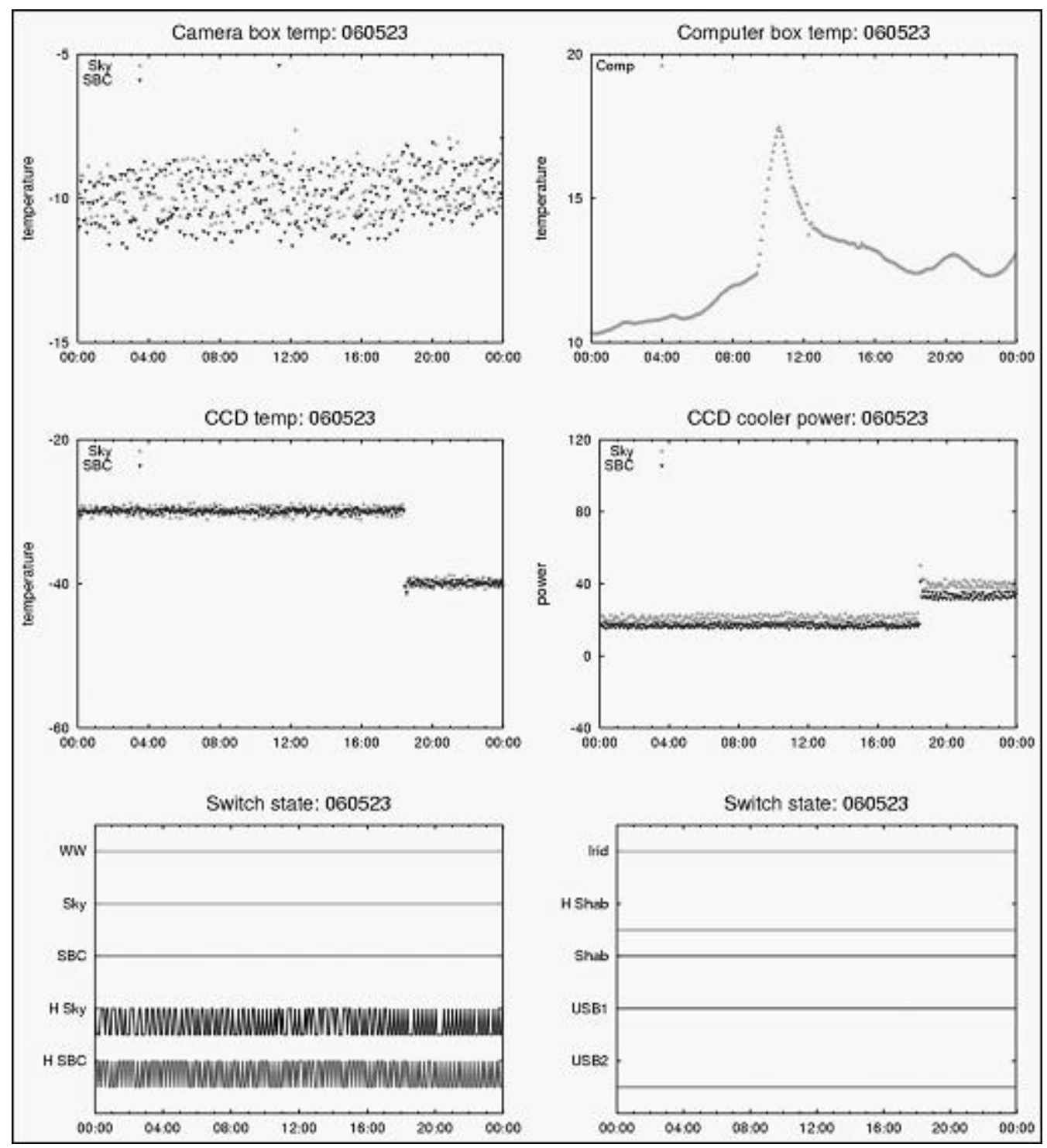

Figure 12: A sample of the status data sent twice a day using the Iridium satellite network from the Gattini supervisor computer the Sydney. During this period the CCD temperature was lowered from $-30^{\circ} \mathrm{C}$ to $-40^{\circ} \mathrm{C}$ with a corresponding rise in CCD peltier cooler power.

- Software (used past experience of UNSW team)

- Cabling and connectors

- Redundancy:- 2 cameras rather than only 1 has been a great feature

The most problematic feature by far to report is icing of the Gattini-SBC glass window, in particular when the temperature dropped dramatically in May. Prior to this little icing of the window occurred. Though we are unsure exactly what is the major cause of the icing the following can be said with certainty (1) Pyrex glass has a high emissivity (0.95) therefore is an excellent radiator of heat (2) Pyrex glass does not transmit NIR wavelengths therefore does not pass heat through from the inside to the outside very well, compared to, for example, Calcium Fluoride (3) a flat piece of glass with a large indent round the rim is not ideal for stopping ice and/or snow accumulating (4) a flat piece of glass sees virtually all of the sky that is at a very low temperature and (5) the highly curved acrylic dome from a geometrical point of view has a lower change of acquiring snow on the outside, or for it to remain very long when it does. 
To remedy the icing issue (that is largely on the inside surface) the Concordia staff along with winter-over astronomer Eric Aristidi constructed a heated cover with an acrylic window, as shown in Figure 17. We are monitoring the progress.
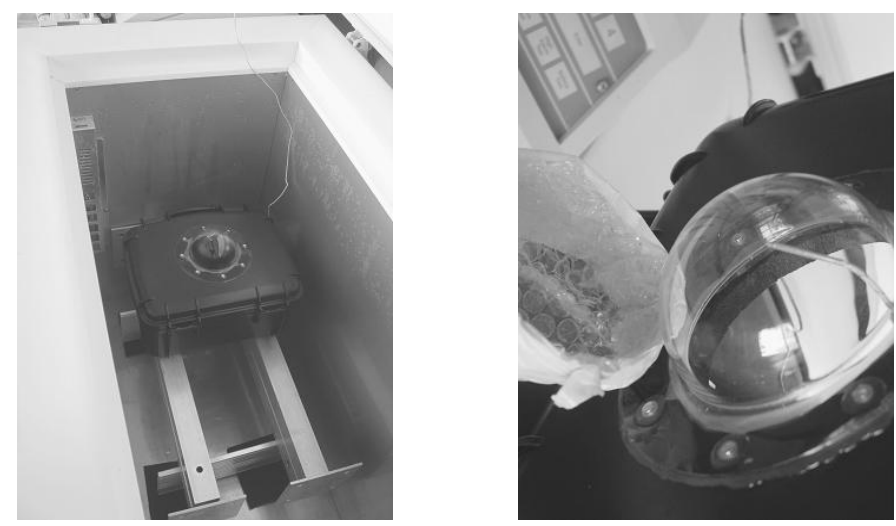

Figure 13: (left) A camera enclosure under low temperature testing. The biological fridge provided a temperature range from ambient (in Sydney) to $-80^{\circ} \mathrm{C}$, quite adequate for the ambient temperatures expected at the Dome $\mathrm{C}$ site.

Figure 14 (right) A Platinum resistance thermometer (PRT) placed in the dome of the Gattini-allsky enclosure during low temperature cycling. Several PRTs were placed inside the enclosure during the cycling to ascertain the level of thermal stratification.

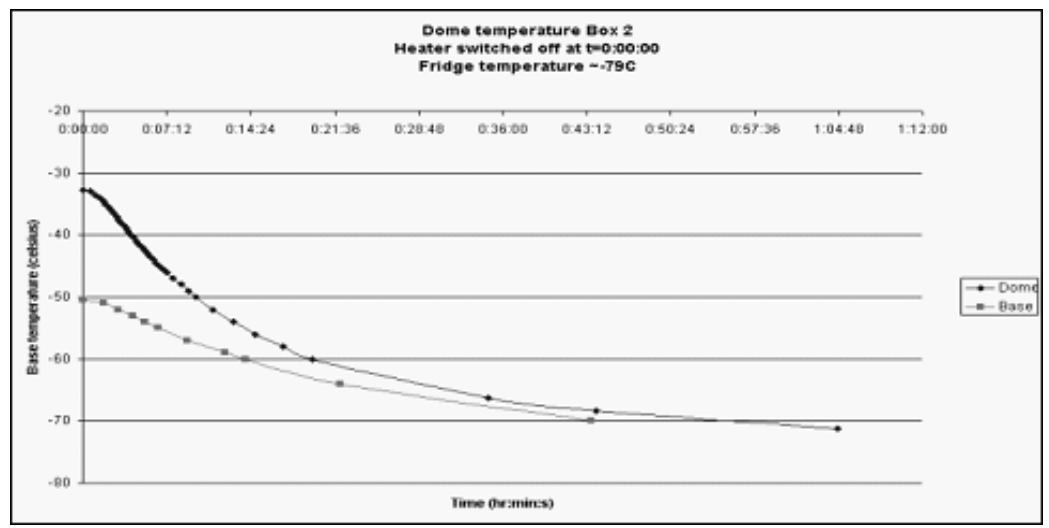

Figure 15: Temperature inside the Gattini-allsky enclosure as a function of time after the internal heaters were switched off at an ambient temperature of $-79^{\circ} \mathrm{C}$. The plots correspond to different height positions within the enclosure and were used to calculate heat loss rate through the acrylic dome (the main source of heat loss). Under normal operation the forced air fans of the CCD Peltier cooler provide enough air circulation to limit the amount of thermal stratification. 

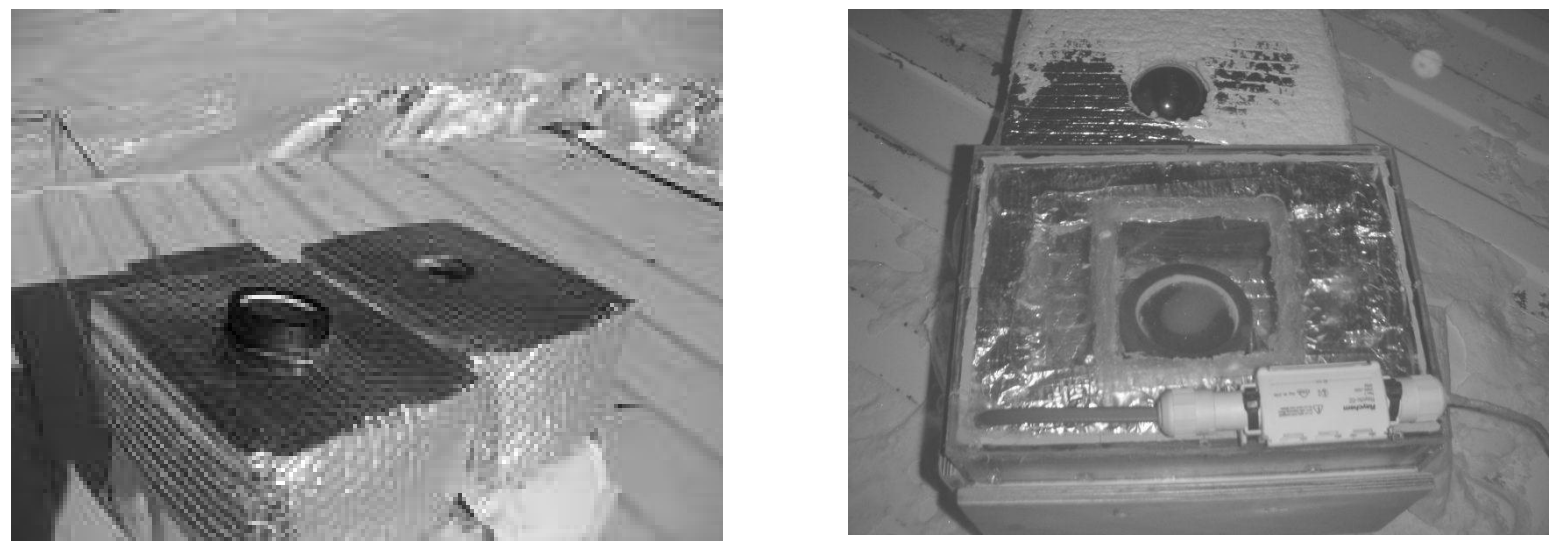

Figure 16: (left) The newly installed Gattini cameras inside their respective insulated enclosures on the roof of the atmospheric laboratory at Dome C. The Gattini-SBC window suffered icing problems from May onwards.

Figure 17: (right) The same enclosures as of 1 week ago. To try and remedy the icing problem a separate enclosure with acrylic window and heating supply was made by Eric Aristidi, 2006/7 winter-over astronomer, and the Concordia staff. Initial pictures taken by Gattini-SBC look promising. Note the absence of ice and/or snow from the acrylic dome compared to the enclosure itself.

\section{PRELIMINARY RESULTS}

The Gattini cameras have been operating since their installation in January 2006. Icing of the Gattini-SBC camera aside the cameras have performed exceptionally well. Thorough data reduction is currently in progress, however, preliminary results from raw data can be presented here.

\subsection{Sky Background variation versus solar and lunar altitude}

Variation of the sky background versus solar altitude is shown in Figure 18 for Gattini-SBC. The y-axis units are median pixel flux value as measured by the $\mathrm{CCD}$ (accurate conversion to $\mathrm{AB}$ magnitude awaits further analysis). For the data plotted distinction is made between observations when the moon is either above or below the horizon. Data reduction on the full data set is required to determine the solar altitude that equates to astronomical dark time at the Dome $\mathrm{C}$ site (usually stated as $-18^{\circ}$ ) though found to be slightly less at high altitude sites such as Paranal [8].

The maximum increase in sky background due to the moon is approximately 50-80 times that of dark sky value.

Equivalent plots for Gattini-allsky are shown in Figure 19. Not intentionally by design the cameras have similar flux gathering power per pixel (the focal length difference is compensated by the difference in pixel area on the sky). Therefore similar maximum values of intensity of the sky background are seen. As expected, due mostly to star contamination, the minimum flux per pixel measured is much higher than that of Gattini-SBC.

\subsection{Cloud Cover}

Cloud cover estimates will be obtained from the images taken by Gattini-allsky. From preliminary analysis the percentage of cloud-free nights appears to be equal or better to the $70 \%$ figure measured by ICECAM [9]. 

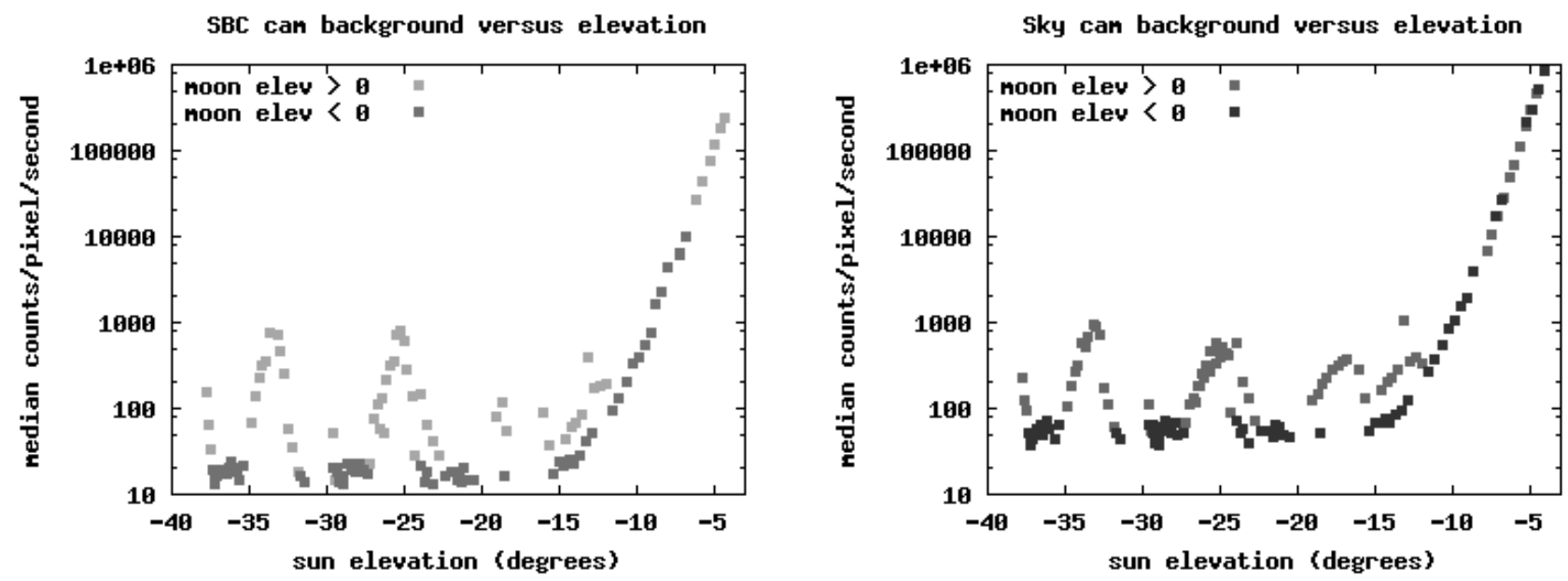

Figure 18: (left) Gattini-SBC raw pixel median count rate versus solar elevation. Distinction is made between measurements taken with a lunar altitude above and below zero. The data shown is calculated from all available images (on average 2 per day since commencement of operation) via the Iridium satellite transfer. This is a factor of 36 times smaller than the number of images stored locally at the site. A thorough data analysis is in progress.

Figure 19: (right) The same plot is shown for Gattini-allsky. See text for details.

\section{CONCLUSIONS}

The automated Gattini cameras are twin site testing instruments that are currently making frequent measurements of the properties of the skies above the Antarctic Dome C. These measurements will be used to investigate (1) optical sky brightness in the sloan g' band during dark time (2) optical sky brightness in the sloan g' band versus solar, lunar altitude and lunar phase (3) optical extinction estimate (4) accurate cloud cover statistics and (5) detection of bright aurora.

We hope for the continued operation of Gattini instruments at Dome C for further 2-3 years or more.

\section{ACKNOWLEDGMENTS}

The authors would like to thank the following people and/or institutions (1) PNRA (Programma Nazionale di Ricerche in Antartide) for amazing logistical support at a timely moment (2) IPEV (Institut Polaire Francais Paul Emile Victor) and PNRA staff assistance at the Concordia station (3) the National Science Foundation for loan of an Iridium card (4) the Australian Antarctic Division and (5) Runa Briguglio, PHD student, University of Roma La Sapienza, for photographic aid at the site prior to shipment.

\section{REFERENCES}

1. Aristidi et al., 2005, A\&A 444..651A

2. Lawrence, J.S., Ashley, M.C.B., Tokovinin, A., Travouillon, T. 2004, Nature, 431, 278

3. Ragazzoni, R., et al. 2005, EAS Publications Series, 14, 161

4. Lawrence, J.S., Burton, M.G., Ashley, M.C.B., \& Storey, J.W.V. 2005, EAS Publications Series, 14, 321

5. Lloyd, J.P., Swain, M., van Belle, G.T., Coude du Foresto, V., Walker, C.K., Traub, W.A., \& Storey, J.W.V.12004, American Astronomical Society Meeting Abstracts, 205, \#181.03

6. Kenyon, S.L., \& Storey, J.W.V.2006, PASP, 118, 489

7. Lawrence, J.S., et al.2003, ESA SP-539: Earths: DARWIN/TPF and the Search for Extrasolar Terrestrial Planets, 497

8. Patat, F. 2004, The Messenger, 115, 18

9. Ashley, M. C. B., Storey, J.W., \& Burton, M.G. 2003, Astronomy in Antarctica, 25th meeting of the IAU, Special Session 2, 18 July, 2003 in Sydney, Australia, meeting abstract, 2 